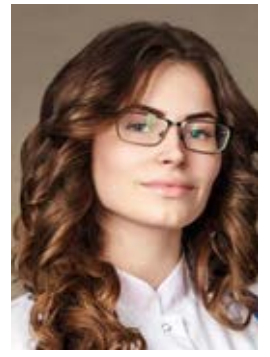

А. Ю. ВениАиктова

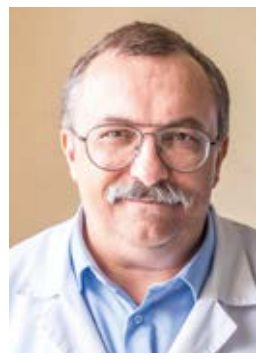

А. В. Борсуков

Немедикаментозное лечение неалкогольной жировой болезни печени: оценка эффективности ультразвуковой стеатометрией

\author{
А. Ю. ВениАиктова, аспирант \\ А. В. Борсуков, А.М.Н., профр., Аиректор
}

Проблемная научно-исслеАовательская ^аборатория «Аиагностические исСАеАОвания и малоинвазивные технологии» ФГБОУ ВО «СМОАенСКИй государственный меАицинский университет» МинзАрава России, г. Смоленск

\title{
Non-drug treatment of non-alcoholic fatty liver disease: evaluation of effectiveness with ultrasound steatometry
}

D. Yu. Venidiktova, A. V. Borsukov

Smolensk State Medical University, Smolensk, Russia

\section{Резюме}

Цель. Оценка эффективности немедикаментозного лечения пациентов с неалкогольной жировой болезнью печени с использованием метоАа количественной ультразвуковой стеатометрии. Материалы и метоАы. Быми обслеАованы 118 пациентов в возрасте 18-75 ^ет, из них 52 (44\%) мужчин и 66 (56\%) женщин по еАиному Аиагностическому алгоритму из четырех этапов: анкетирование и сбор жалоб, оценка качества жизни кАинический осмотр, неинвазивная биоимпеАансометрия с послеАующей оценкой инАекса массы жира, Ультразвуковое исслеАование печени В В-режиме, количественная ультразвуковая стеатометрия посреАством оценки коэффициента затухания ультразвуковой волны. Пациентам назначена инАивиАуаАьная немеАикаментозная терапия в виАе инАивиАуаАьной программы лечебного питания и Аозированных физических нагрузок. Аинамическая оценка снижения Аоли жировой ткани и выраженности жировой инфияьтрации печени проводилась в точках 6, 12, 18, 24 месяца. Биопсия печени с послеАующим гистологическим анализом по шкале SAF выполнена у 32 пациентов. Результаты. Аля оценки эффективности немеАикаментозного ^ечения У пациентов производимось наблюАение за тенАенцией снижения Аолей соАержания жира и выраженности жировой инфимьтрации в печени. Все пациенты были разАелены на Аве группы согласно уровню их комплаентности к назначенному лечению. Снижение Аоли жировой ткани у пациентов с высоким уровнем комп^аентности - АО 76,2\%, у пациентов с низким уровнем комплаентности АО $89,1 \%$ от исхоАных значений. Снижение жировой инфимьтрации печени у пациентов С высоким уровнем комп^аентности-АО $80,2 \%$, у пациентов с низким Уровнем компАаентности - АО 92, 1 \% от исхоАноГО. ЗаКАЮчеНИе УАьтразвуковая стеатометрия - информативный метоА ААя Аинамиче ского мониторинга неалкогомьной жировой болезни печени в рамках проведения немеАикаментозной терапии. Корреляция Аанных количественной ультразвуковой Аиагностики стеатоза с биопсией на стаАии SO СоОтветствует $r=0,85$; на стаАии $S 1-r=0,72, S 2-r=0,73$, на стаАии $S 3-r=$ 0,84, что свиАетельствУет о высокой информативности Аанного метоАа. Ключевые Слова: неалкогольная жировая болезнь печени, ультразвуковая стеатометрия.

\section{Summary}

Objective. Evaluation of the effectiveness of non-drug treatment in patients with non-alcoholic fatty liver disease using the method of quantitative ultrasound steatometry. Material and methods. We examined 118 patients aged $18-75$ years, including 52 (44\%) men and 66 (56\%) women using a single diagnostic algorithm of four stages: questioning and collecting complaints, assessing the quality of life, clinical examination, non-invasive bioimpedansometry, followed by assessment of fat mass index, ultrasound examination of the liver in the B-mode, quantitative ultrasound steatometry by evaluating the attenuation coefficient of the ultrasonic wave. Individual non-drug therapy is prescribed to patients in the form of an individual program of therapeutic nutrition and physical activity. A dynamic assessment of the decrease in the proportion of adipose tissue and the severity of fatty liver infiltration was carried out at points of 6, 12, 18, 24 months. A liver biopsy followed by histological analysis on an SAF scale was performed in 32 patients. Results. To assess the effectiveness of non-drug treatment in patients, a tendency of reducing the proportion of fat content and the severity of fatty infiltration in the liver was observed. All patients were divided into two groups according to their level of compliance with the prescribed treatment. Decrease in the proportion of adipose tissue in patients with a high level of compliance - up to $76.2 \%$, in patients with a low level of compliance - up to $89.1 \%$ of the initial values. Decrease in fatty liver infiltration in patients with a high level of compliance - up to $80.2 \%$, in patients with a low level of compliance - up to $92.1 \%$ of the initial one. Conclusion. Ultrasound steatometry is an informative method for non-alcoholic fatty liver disease screening. The correlation of quantitative ultrasound diagnostics data for steatosis with a biopsy at stage SO corresponds to $r=0.85$; at stage $S 1 r=0.72$, at $S 2 r=0.73$, at stage $S 3$ corresponds to $r=0.84$, which indicates the high informativity of this method.

Key words: non-alcoholic fatty liver disease, ultrasound steatometry.

\section{Введение}

Ожирение в настоящее время представляет собой глобальную эпидемию, которой все чаще заболевают лица социально активной, трудоспособной возрастной группы (25-60 лет). Распространенность ожирения в разных странах составляет 23-50\% [7, 2]. Значимость проблемы ожирения определяется угрозой инвалидизации пациентов молодого возраста и снижением общей продолжительности жизни в связи с частым развитием тяжелых сопутствующих заболеваний. Одним из серьезных проявлений ожирения является неалкогольная жировая болезнь печени (НАЖБП): стеатоз и стеатогепатит с возможным развитием последующих изменений: фиброз, цирроз (в том числе его осложнения), гепатоцеллюлярная карцинома $[1,3]$. Качество жизни пациентов с избыточной массой тела снижается в зависимости от длительности и прогрессирования болезни, а также при наличии сопутствующих заболеваний и метаболических осложнений. Ввиду широкого спектра патологических изменений, связанных с наличием ожирения, имеется острая необходимость в поиске оптимального и наиболее эффективного диагностического комплекса для 


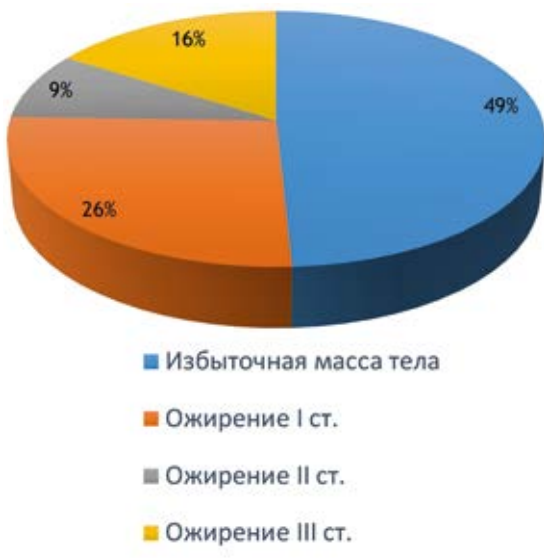

Рисунок 1. Распрелеление пациентов по инАексу массы жира по результатам провеАения неинвазивной биоимпедансометрии.

оценки выраженности подкожного и висцерального жира, распределения жировой ткани, наличия, характера и степени выраженности жирового гепатоза, а также для оценки эффективности немедикаментозной терапии у данных пациентов.

Целью исследования является оценка эффективности немедикаментозного лечения у пациентов с неалкогольной жировой болезнью печени с использованием метода количественной ультразвуковой стеатометрии.

\section{Материалы и методы}

В 2017-2019 годах на базе проблемной научно-исследовательской лаборатории «Диагностические исследования и малоинвазивные технологии» СГМУ (г. Смоленск) были исследованы 118 пациентов многопрофильного стационара в возрасте 18-75 лет (средний возраст - 47,00 \pm 4,85 года), из них 52 (44\%) мужчины и 66 (56\%) женщин.

Критерии включения: согласие на участие в исследовании; возраст старше 18 лет; более $33 \%$ жировой ткани у женщин и более $20 \%$ - у мужчин, по данным неинвазивной биоимпедансометрии (нБИМ); отсутствие регулярного приема лекарственных препаратов; употребление алкоголя не более 30 г в день для мужчин и 20 г в день для женщин (менее 2 баллов по опроснику CAGE); наличие жировой инфильтрации паренхимы печени (менее $50 \mathrm{HU}$ ), по данным анализа мультиспиральной компьютерной томографии (МСКТ) органов брюшной полости, выполненной в плановом

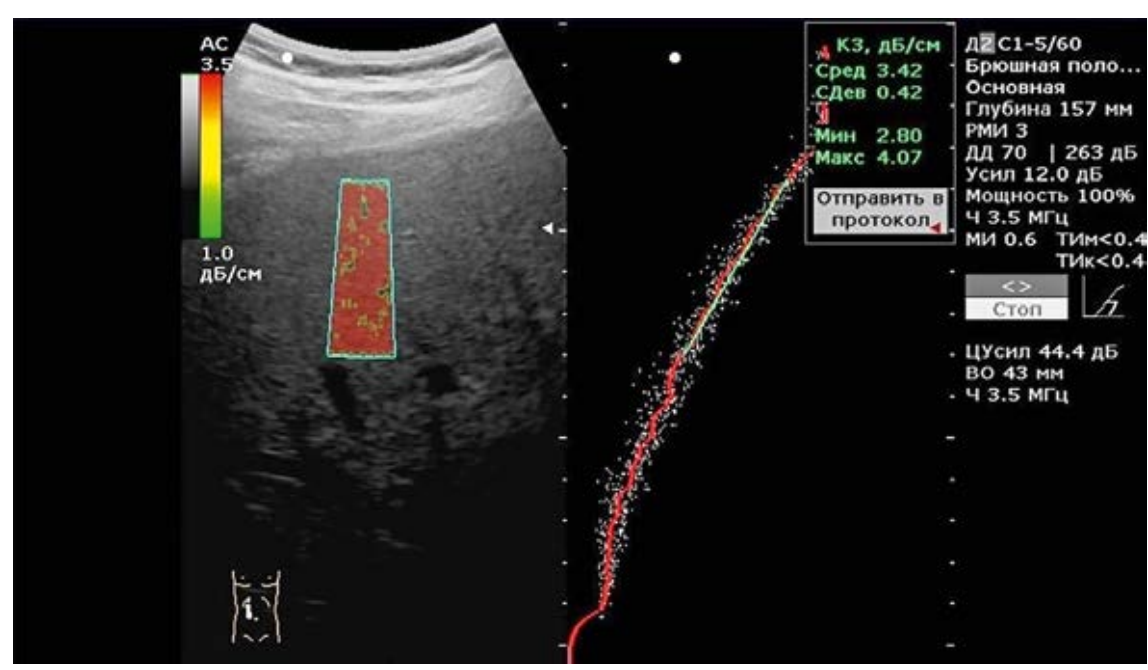

Рисунок 2. УЗ-метол количественного определения стеатоза печени путем определения коэфорициента затухания У3-волны ( $\Delta$ Б/см). Пациентка Н., 43 года, стеатоз печени S3 - коэорорициент затухания равен 3,42 $\pm 0,42 \Delta Б / с$. порядке по решению лечащего врача в рамках дополнительной диагностики основного заболевания.

Критерии невключения: отказ пациента от участия в исследовании; наличие жировой ткани менее $33 \%$ жировой ткани у женщин и менее $20 \%$ - у мужчин, по данным неинвазивной биоимпедансометрии; регулярный прием лекарств; употребление алкоголя выше установленной CAGE) [9]; плотность паренхимы печени менее $50 \mathrm{HU}$, по данным МСКТ.

Все пациенты были исследованы по единому диагностическому алгоритму, который включал в себя четыре этапа.

1. Выявление жалоб, которые свидетельствуют о наличии заболевания печени, сопутствующей патологии нормы (более 1 балла по опроснику органов и систем органов, сахарного диабета первого и второго типа, отягощенного семейного анамнеза, о характере питания и употребления алкогольных напитков (с использованием скрининговой методики оценки хронической алкогольной интоксикации CAGE).

2. Клинический осмотр пациента с оценкой роста (см), веса (кг), индекса массы тела (кг/м²), объема талии (см), объема бедер (см), вычисление их соотношения.

3. Неинвазивная биоимпедансометрия (Omron BF212 [HBF-212-EW, Китай]) с оценкой выраженности жирового сектора организма (\%) и последующим определением индекса массы жира и распределением пациентов на группы с учетом их возраста (различные

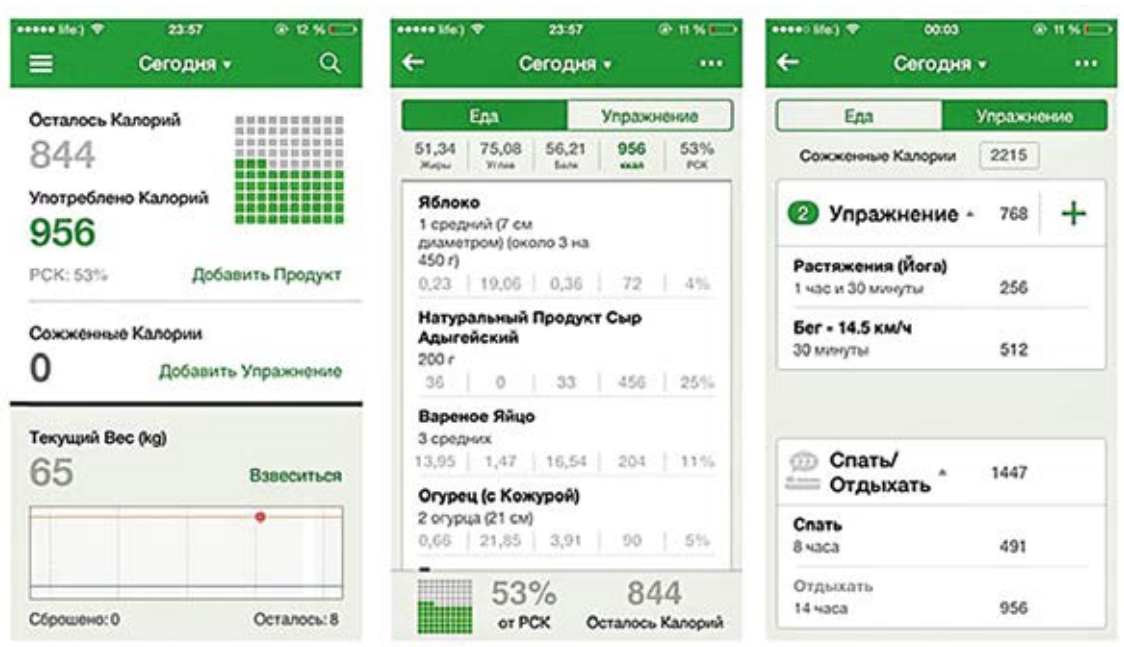

Рисунок 3. Электронный Аневник питания и фризической активности FatSecret. 
Таблица

КАинико-Аиагностическая характеристика выборки на момент набора пациентов в исслеАование

\begin{tabular}{|c|c|c|c|c|c|c|}
\hline \multirow[t]{2}{*}{ Параметр } & \multicolumn{2}{|c|}{ Стеатоз выявмен (S1-S3)* } & \multicolumn{2}{|c|}{ Стеатоз отсутствует (SO)* } & \multicolumn{2}{|c|}{ Bcero } \\
\hline & $\begin{array}{l}\text { Абсолютное } \\
\text { число, } n\end{array}$ & $\begin{array}{l}\text { Относительный } \\
\text { процент }\end{array}$ & $\begin{array}{l}\text { Абсолютное } \\
\text { число, } n\end{array}$ & $\begin{array}{l}\text { Относительный } \\
\text { процент }\end{array}$ & $\begin{array}{l}\text { Абсолютное } \\
\text { число, } n\end{array}$ & $\begin{array}{l}\text { Относительный } \\
\text { процент }\end{array}$ \\
\hline \multicolumn{7}{|c|}{ Пол } \\
\hline Женщины & 64 & 54,3 & 2 & 1,7 & 66 & 56 \\
\hline Мужчины & 50 & 42,3 & 2 & 1,7 & 52 & 44 \\
\hline \multicolumn{7}{|c|}{ Аоля жировой ткани в организме, по Аанным неинвазивной биоимпеАансометрии, \% } \\
\hline $\begin{array}{l}\text { Более } 33 \% \text { жировой ткани у женщин, } \\
\text { Более } 20 \% \text { жировой ткани у мужчин }\end{array}$ & 114 & 96,6 & 4 & 3,4 & 118 & 100 \\
\hline \multicolumn{7}{|c|}{ Жировая инфимьтрация паренхимы печени, по Аанным УзИ печени в В-режиме } \\
\hline І степень & 30 & 25,4 & 0 & 0 & 30 & 25,4 \\
\hline ॥ степень & 42 & 35,6 & 4 & 3,4 & 46 & 39,0 \\
\hline
\end{tabular}

Примечание: *-по данным количественной ультразвуковой стеатометрии печени.

показатели индекса массы жира у пациентов в возрасте $18-37$, 38-57, 58-75 лет): первая группа избыточная масса жира ( $\mathrm{n}=58$; 49,2\%), вторая группа - ожирение первой степени $(\mathrm{n}=31 ; 26,3 \%)$, третья группа - ожирение второй степени $(\mathrm{n}=10 ; 8,5 \%)$, четвертая группа - ожирение третьей степени $(\mathrm{n}=19 ; 16,0 \%)$ (рис. 1$)$.

4. Ультразвуковое исследование печени (Hitachi Preirus, Япония) в В-режиме с оценкой размеров правой и левой долей печени (мм), эхогенности, звукопроводимости, визуализации крупных сосудов и желчных протоков с определением выраженности жировой инфильтрации печени [4]. Количественная ультразвуковая стеатометрия печени («БИОСС Ангиодин Соно-П/
Ультра», Россия) с визуальной и количественной оценками коэффициента затухания ультразвуковой волны (дБ/см) (рис. 2). [8].

Для определения выраженности стеатоза использовалась шкала (дБ/ см), коррелирующая со степенью стеатоза, по данным биопсии печени: $\mathrm{S} 0$ - нет стеатоза (менее 2,19 дБ/см); $\mathrm{S} 1$ - минимальный стеатоз, менее $5 \%$ гепатоцитов со стеатозом (2,2-2,29 дБ/ см); S2 - умеренный стеатоз, менее 6-32\% гепатоцитов со стеатозом (2,32,9 дБ/см); S3 - выраженный стеатоз, 33-100\% гепатоцитов со стеатозом (более 2,9 дБ/см).

32 пациентам была выполнена биопсия печени с ультразвуковой навигацией с последующим анализом гистологического препарата по шкале

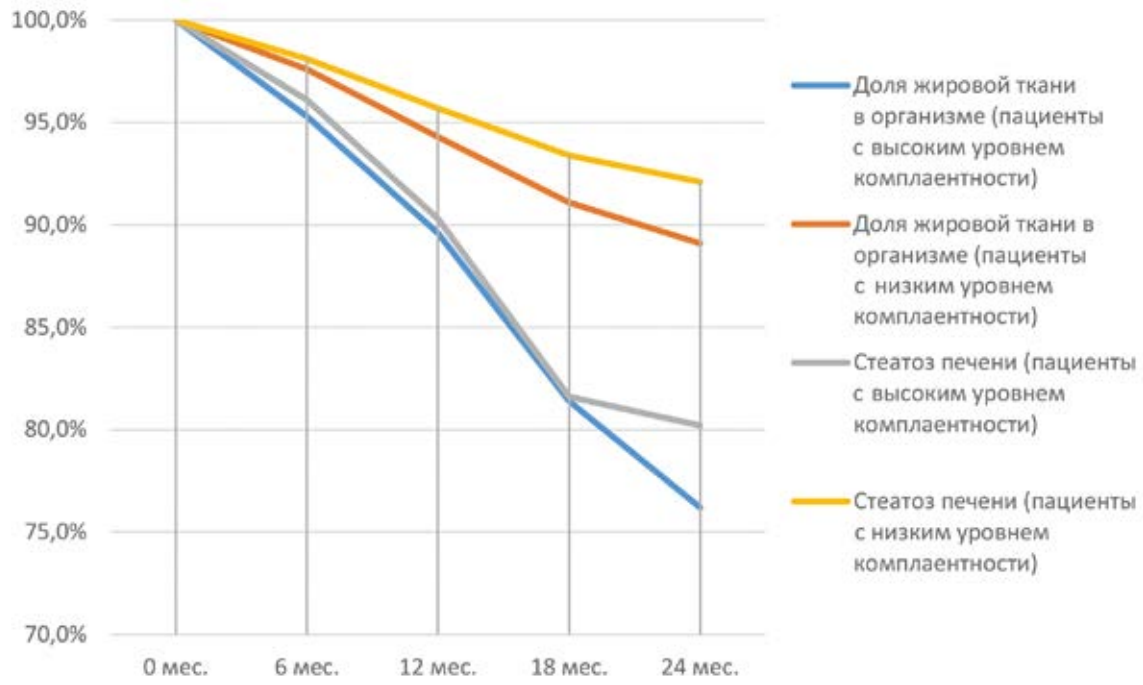

Рисунок 4. Тенденция снижения массы жира и изменения жировой инорильтрации в органе у пациентов исследуемой группы ( $n=118)$ на протяжении 24 месяцев.

SAF с определением выраженности жировой инфильтрации, воспалительного процесса, фиброза [9].

После прохождения комплексного диагностического алгоритма при первом визите каждому пациенту индивидуально была подобрана немедикаментозная терапия (рациональное питание и физическая нагрузка, направленные на снижение массы тела) на основании индекса массы жира, наличия (отсутствия) метаболического синдрома, наличия (отсутствия) сопутствующих заболеваний $[5,10]$.

Индивидуальная суточная потребность килокалорий рассчитывалась вручную посредством умножения скорости основного обмена на коэффициент физической активности. Из индивидуальной суточной потребности килокалорий у каждого пациента вычиталось 500 ккал, в итоге было получено числовое значение суточной калорийности рациона пациента. Целевой показатель потери веса - 2-4 кг в месяц, 7-10\% от общей массы тела в 12-месячный период (как предиктор значительного уменьшения стеатоза печени). Потребление жидкости - из расчета 30 мл на 1 кг веса. Также с пациентами были проведена мотивационная беседа с акцентированием необходимости рационального питания для предотвращения развития осложнений НАЖБП. При наличии у пациента сопутствующих заболеваний производилась индивидуальная коррекция питания совместно с лечащим врачом (диеты № 2, 4, 5, 10). 
Физическая активность подбиралась для каждого пациента индивидуально с учетом его возраста, пола, роста, веса, процентного содержания жировой ткани в организме, сопутствующих заболеваний, субъективной готовности выполнять ту или иную физическую нагрузку и подразделялась на базовую и дополнительную.

Всем пациентам были объяснены особенности использования электронного дневника питания и физической активности FatSecret, с помощью которого производилось текущее динамическое наблюдение вне исследовательского центра с применением пошаговой коррекции терапии в случае необходимости (рис. 3).

Весь диагностический четырехэтапный алгоритм повторялся на каждом из пяти очных визитов пациентов $(0,6,12,18,24$ месяца).

В каждой возрастной и гендерной группе на каждом очном этапе была проведена оценка уровня комплаентности пациентов к соблюдению всех рекомендаций врача относительно немедикаментозной терапии.

При статистической обработке данных с определением корреляционной связи выдвигались следующие статистические гипотезы: Н0 - при попарном сравнении информативность методов (количество пациентов, у которых были получены идентичные данные, идентичные гистологическому) одинаковая в одинаковые временные периоды; Н1 - информативность методов значимо отличается. Результаты попарного сравнения информативности методов на разных этапах наблюдения за пациентом считались статистически значимыми при $\mathrm{p}<0,05$.

\section{Результаты и их обсуждение}

Прогресс снижения массы жира (согласно определенной доле жировой ткани в процентах, по данным неинвазивной биоимпедансометрии) и изменения жировой инфильтрации в органе (по данным количественной ультразвуковой стеатометрии) на протяжении 24 месяцев представлены на рис. 4.

Корреляция данных проведенной на этапе набора пациентов в исследование количественной ультразву-

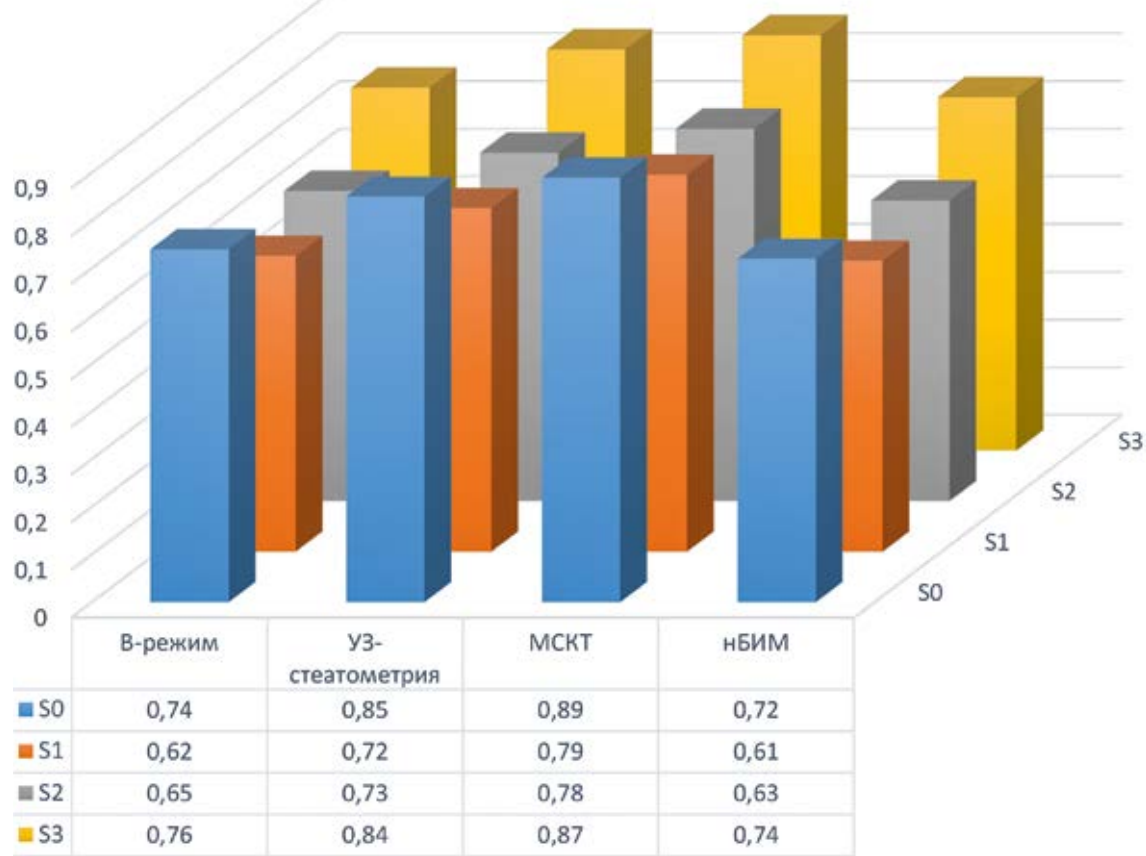

Рисунок 5. Корреляция результатов различных методов Аиагностики с гистологическим сталированием стеатоза печени у пациентов с НАЖБП на этапе набора в исслеАование.

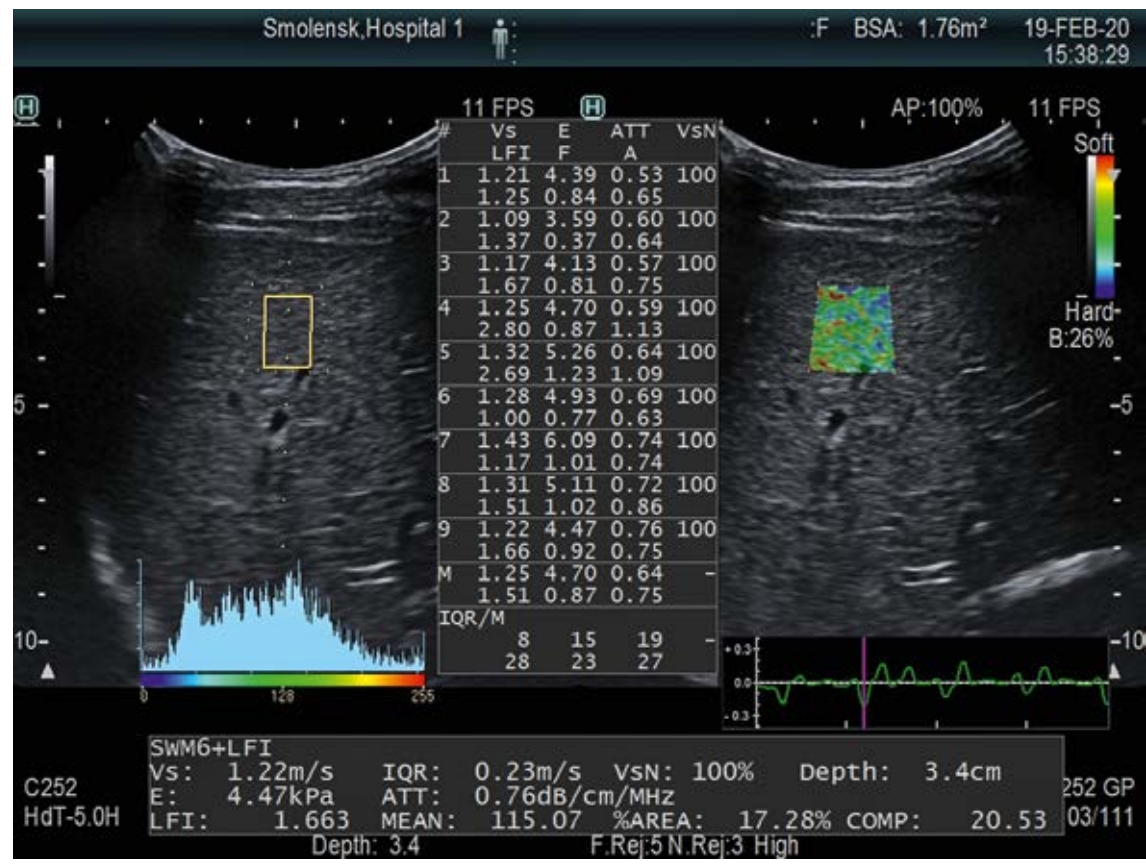

Рисунок 6. У3-метод количественного определения стеатоза печени путем определения коэффри циента затухания УЗ-волны (АБ/см/МГц) в рамках комп^ексной технологии комбинированной эластографиии. Пациентка В., 28 ^ет, стеатоз печени, S1-S2 - коэфофициент затухания - 0,64 АБ/см/МГц, воспалительная активность АО - инАекс 0,75, ффиброзные изменения F0 - инАекС 0,87 (уровень АОстоверности полученных результатов с учетом рекомендуемой метолики проведения исслеАования - 100\%).

ковой диагностики стеатоза печени (до начала немедикаментозной терапии) с биопсией (рис. 5) на стадии $\mathrm{S} 0$ и $\mathrm{S} 3$ соответствует $\mathrm{r}=0,85, \mathrm{r}=0,84$, что свидетельствует о высокой информативности данного метода и возможности замены гистологического исследования у пациентов с избыточной массой жира на определение ко- эффициента затухания ультразвуковой волны в тканях, в том числе в процессе динамического мониторинга.

В настоящее время внедряется ультразвуковой метод комбинированной эластографии (интеграция двух методов для неинвазивной оценки жесткости печени: технологии компрессионной эластографии, совмещенной 
с эластометрией сдвиговой волны) с последующим определением индекca F (фиброза) и индекса А (степени воспаления). Одновременно данная методика позволяет провести количественную оценку жировой инфильтрации органа с определением показателя ATT (индекса затухания) в дБ/см/МГц (рис. 6). Преимуществом метода является возможность оценить фиброз и стеатоз печени вне зависимости от наличия воспаления и холестаза [6].

С использованием данного комбинированного метода проведена неинвазивная оценка диффузных изменений печени у 49 пациентов исследуемой группы в рамках очных визитов в исследовательский центр. Данные по жировой инфильтрации органа сопоставимы с показателями, полученными у всех пациентов методом оценки коэффициента затухания ультразвуковой волны в тканях в 96\% случаев $(\mathrm{n}=47)$.

\section{Выводы}

1. Использование комплексного ультразвукового диагностического алгоритма у пациентов с неалкогольной жировой болезни печени позволяет оценивать выраженность стеатоза печени на этапах динамического мониторинга.

2. Количественная ультразвуковая стеатометрия - информативный метод оценки выраженности неалкогольной жировой болезни печени у пациентов с избыточной массой тела до, после и во время немедикаментозного лечения.

\section{Список митературы}

3. Chalasani N., Younossi Z., Lavine J.E. et al. The diagnosis and management of non-alcoholic fatty liver disease: practice guideline by the American Gastroenterological Association, American Association for the Study of Liver Diseases, and American College of Gastroenterology. Gastroenterology 2012; 142: 1592-609.

4. European Association for the Study of the Liver (EASL); European Association for the Study of Diabetes (EASD): European Association for the Study of Obesity (EASO). EASL-EASD-EASO Clinical Practice
Guidelines for the management of non-alcoholic fatty liver disease. J. Hepatol. 20 16; 64 (6): 1388-402. DOI: 10.1016/j.jhep.2015.11.004.

5. La Brecque, Douglas R, etc. World Gastroenterology Organisation Global Guidelines. Nonalcoholic Fatty Liver Disease and Nonalcoholic Steatohepatitis. Journal of Clinical Gastroenterology: July 2014.- Volume 48.- Issue 6.- P. 467-473.

6. Schwenzer N. F., Springer F., Schraml C., Stefan N. Machann J., Schick F. Non-invasive assessment and quantification of liver steatosis by ultrasound, computed tomography and magnetic resonance // J Hepatol 2009; 51: 433-45.

7. Simona Leoni, Francesco Tovoli, Lucia Napoli, Ilaria Serio, Silvia Ferri, Luigi Bolondi. Current guidelines for the management of non-alcoholic fatty liver disease: A systematic review with comparative analysis. World A systematic review with comparative analysis. Worlo
J Gastroenterol. 2018 Aug 14; 24 (30): 336 I-3373.

8. Yada N, Tamaki N, Koizumi Y, Hirooka M, Nakashima O. Hiasa Y, Izumi N, Kudo M.: Diagnosis of fibrosis and activity by a combined use of strain and shear and activity by a combined use of strain and shear Dis, 2017; 35(6): 515-520. DOI: 10.1159/000480140.

9. Балукова Е.В., Барышникова Н.В., Белоусова А.Н. Неалкогольная жировая болезнь печени: современное состояние проблемы // Фарматека. 2016; 2: 63-8.

10. Борсуков А. В., Венидиктова А. Ю. УАьтразвуковая Аиагностика стеатоза печени: разработка новой методики количественной оценки патологического процесса // МеАицинский алффавит. 2017. 19. С. 47-51.

11. Ивашкин В.Т., Маевская М. В., Жаркова М.С. илр. Алгоритмы Аиагностики и лечения в гепатологии. Москва. МЕАпресС-инорорм. 2016. С. 155.

12. Кошурникова А.С., Аукина Е.В. Ожирениеиметаболический синАром: вопросы Аиагностики профрилактики и лечения // ^ечащий врач. 2017. № 10. С. 66.
Аля цитирования: Венидиктова А. Ю., Борсуков А. В. Немедикаментозное лечение неалкогольной жировой болезни печени: оценка эфорективности ультразвуковой стеатометрией.. МеАицинский алфавит. 2020; (10): 38-42. https://doi. org/10.33667/2078-5631-2020-10-38-42
For citation: Venidiktova D. Yu., Borsukov A.V. Non-drug treatment of non-alcoholic fatty liver disease: evaluation of effectiveness with ultrasound steatometry. Medical alphabet. 2020: (10): 38-42. https://doi.org/10.33667/2078-5631-2020-10-38-42

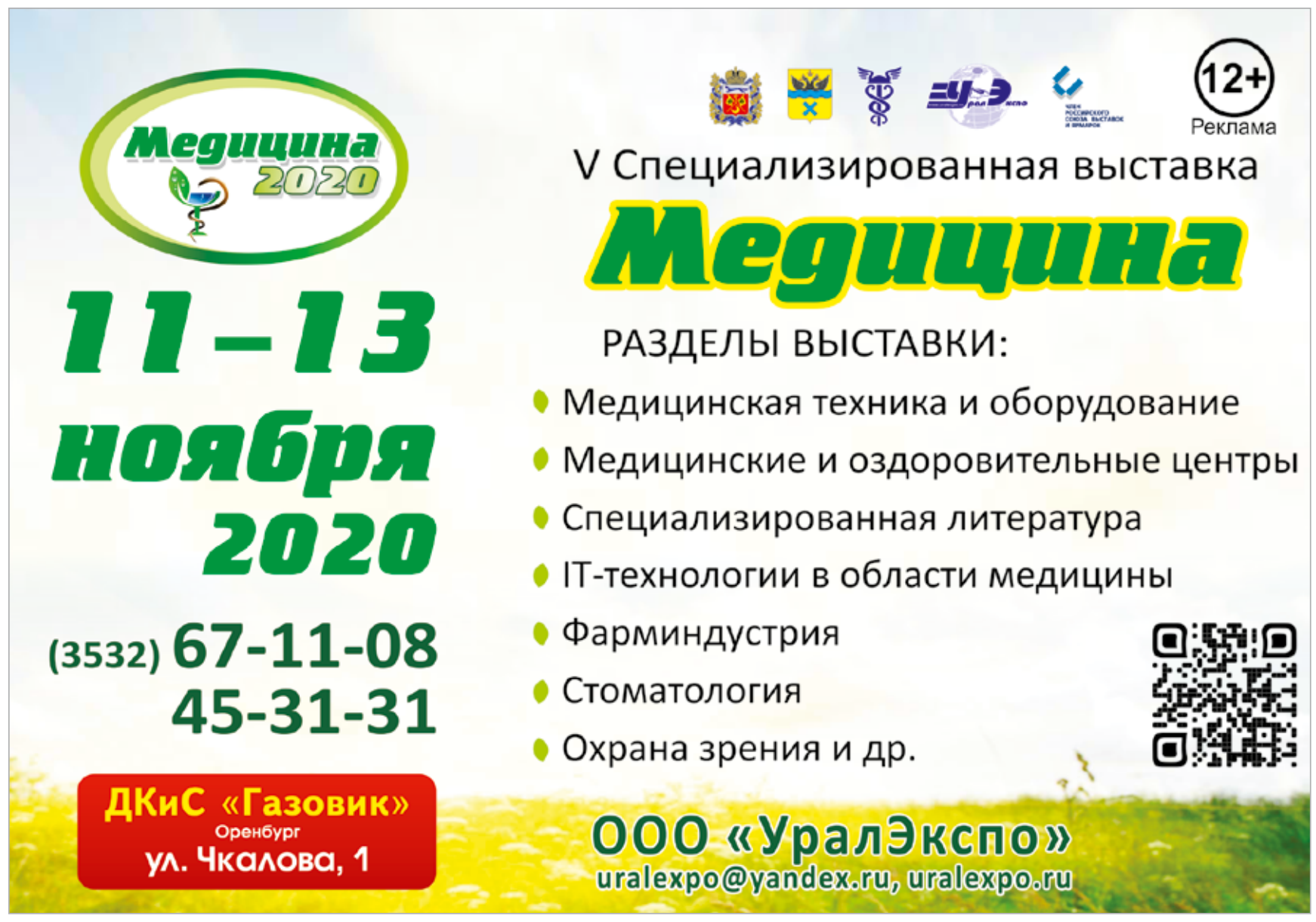

